\title{
Efficiency of Universal Back Resting Device in Public Health Dentistry Outreach Activities: A Cross-sectional Study among Patients and Service Care Providers
}

\author{
Ashna M Benny¹, Senthil Murugappan²
}

\begin{abstract}
Aims and objectives: This study was undertaken to analyze the benefits and drawbacks of the universal back resting device (UBRD) and to use the obtained data to rectify defects if any and modify it for the betterment of treatment outcomes at camp sites.

Materials and methods: This is a cross-sectional questionnaire study undertaken among a group of 100 patients and 100 healthcare providers who have received treatment with the aid of and provided treatment with the UBRD, respectively. The data that were obtained by analyzed and percentages were calculated using SPSS 17 for windows.

Results: The results of this study conclusively pointed out that the UBRD meets the demands that it was intended for and it can also be used as an alternative to portable dental chairs at camp sites or even in primary health centers when the available funds are insufficient in ratio to the number of people requiring treatment. The possible alteration that can be made to the device to increase its efficiency was also noted.

Conclusion: The UBRD has proved to be useful in providing oral healthcare at camp sites and could be stated that it is a fair enough alternative to conventional chairs when minimum resources are available.

Clinical significance: The UBRD is a unique device that can aid in providing better treatment and is cost efficient and if it is implemented in camp/outreach sites, it would drastically increase the number of patients getting treated in a short period of time and to an efficiency level almost as equal as a portable dental chair.

Keywords: Camps, Dental, Efficiency, Outreach programs, Universal back resting device.

Journal of Scientific Dentistry (2020): 10.5005/jp-journals-10083-0933
\end{abstract}

\section{INTRODUCTION}

Helping the people in our community who cannot afford oral healthcare has always been an important part of the mission and service of Public Health Dentistry. We bring much needed dental care to people who might not otherwise have access to it. Focusing on rendering quality healthcare in areas with below average availability of resources is challenging. For millions of Indians who suffer from dental ailments, consulting a dental surgeon remains an elusive dream, especially in rural areas. High capital required to establish dental operatories in rural outposts, i.e., primary health centers (PHCs) and during outreach programs is an obvious impediment. ${ }^{1}$ Dental vans owned by teaching institutions and philanthropic organizations have limited operational capability in rural terrain due to lack of motorable roads. Portable dental units though are an alternative, they are costly. The basic portable dental unit includes an operator light source, an examination kit, a portable head rest, and a first-aid kit for dental purposes. These portable dental units used have a rotary instrument and an operator light fixture that is packed in two cases. More complex portable units include a vacuum canister, ultrasonic scaler, radiographic equipment along with compressors for air-water syringes, and high and low speed handpieces. This equipment is stored and transported in durable boxes and cases. ${ }^{2,3}$ While we have provisions for dental materials, instruments, and ultrasonic scalers, transporting dental chairs in large numbers always poses a problem.

To overcome this issue, the Department of Public Health Dentistry of Indira Gandhi Institute of Dental Sciences (IGIDS),
${ }^{1}$ Department of Periodontia, Sree Mookambika Institute of Dental Sciences, Kulasekharam, Tamil Nadu, India

${ }^{2}$ Department of Public Health Dentistry, Indira Gandhi Institute of Dental Sciences, Sri Balaji Vidyapeeth, Puducherry, India

Corresponding Author: Senthil Murugappan, Department of Public Health Dentistry, Indira Gandhi Institute of Dental Sciences, Sri Balaji Vidyapeeth, Puducherry, India, Phone: +91 9486365954, e-mail: senthilm@igids.ac.in

How to cite this article: Benny AM, Murugappan S. Efficiency of Universal Back Resting Device in Public Health Dentistry Outreach Activities: A Cross-sectional Study among Patients and Service Care Providers. J Sci Dent 2020;10(2):34-37.

Source of support: Nil

Conflict of interest: None

Sri Balaji Vidyapeeth, Puducherry has designed the universal back resting device (UBRD). It comprises headrest with locking hooks and hooking device that can be adjusted in three steps to enable the neck movement for the patient, velcro fastener to fix the device firmly in the back of the chair and a bottom section to reduce the height of the device (Fig. 1).

The UBRD is portable, compact, light-weight, can be fixed to any chair, versatile, economical, and does not require a skilled person to be put to use. The device is one of its kind and has enabled dentists to work with better efficiency and has also aided in the comfort of patients. The device has been patented and this study has been undertaken to analyze the efficiency of the device from the perspective of both the dentists and the patient. 


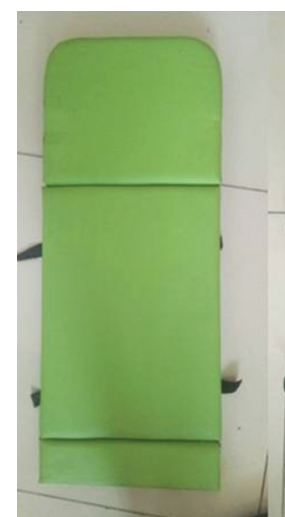

Front view

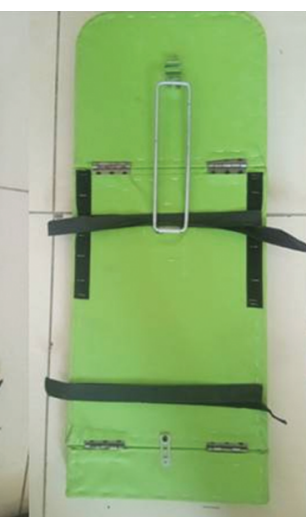

Back view

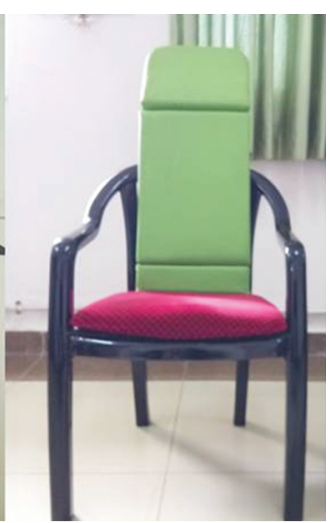

Mounted view
Fig. 1: Universal back resting device

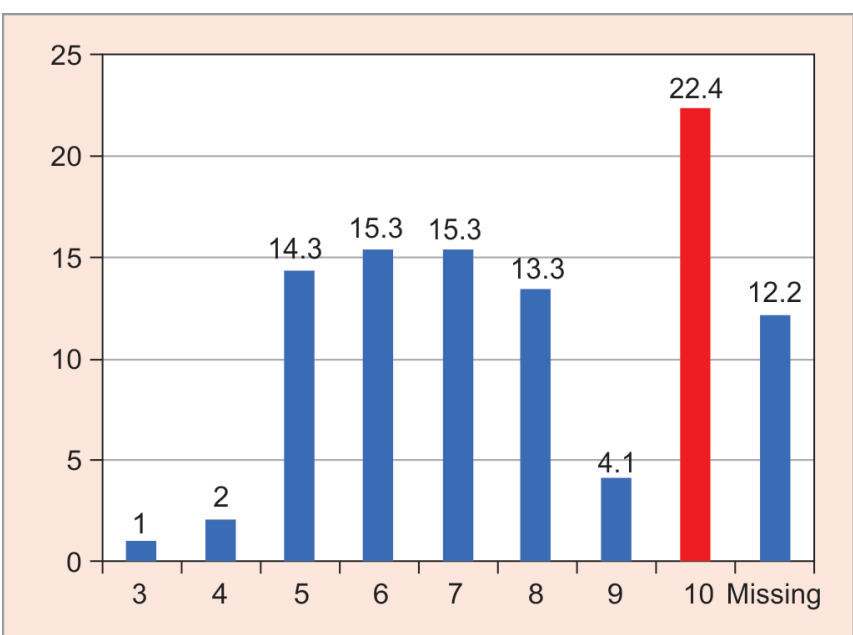

Fig. 3: Perception of patients undergoing treatment at camp sites. How would you rate your ability to cooperate to the procedure by the dentist as with the conventional chair on a scale of 1-10?

\section{Aims and OBjectives}

This study was undertaken to analyze the benefits and drawbacks of the UBRD and to use the obtained data to rectify defects if any and modify it for the betterment of treatment outcomes at camp sites.

\section{Materials and Methods}

This questionnaire study is undertaken to analyze the efficiency of the UBRD from the perspective of both the dentists and the patient. The sample population included 100 healthcare providers of IGIDS and 100 patients who have been treated using this device while at treatment camps. The inclusion criteria were that the healthcare providers should have attended at least two treatment camps and worked with the UBRD. The population who have not attended treatment camps or have attended less than two were excluded from the study. A 11-point questionnaire according to Likert scale was given for the healthcare providers and a 6-point questionnaire for the patients. A pilot study was performed for a sample population of 30 . The questionnaire was analyzed and results concluded in frequency distribution, percentages were calculated using SPSS 17 for windows.

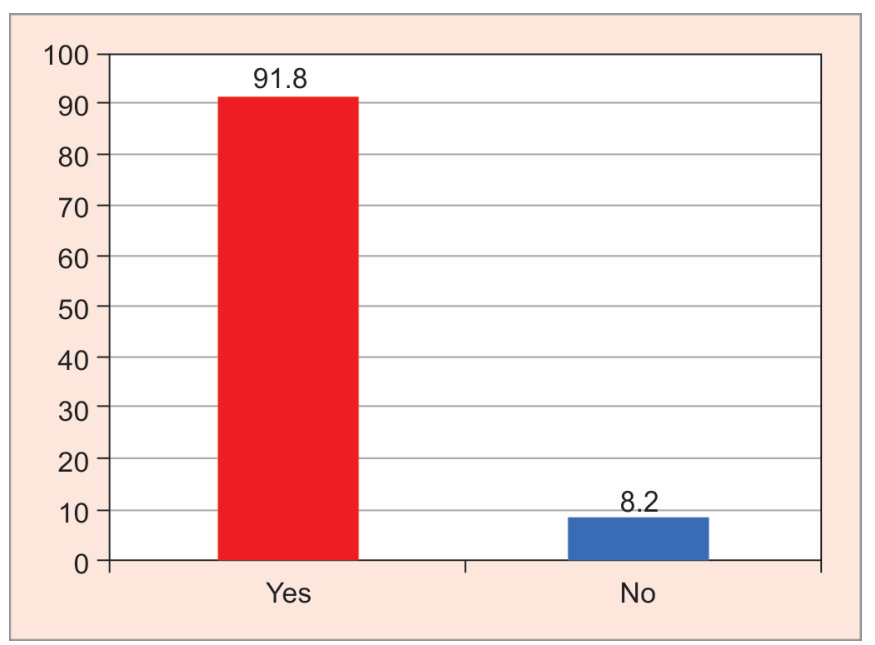

Fig. 2: Perception of patients undergoing treatment at camp sites. Do you experience any physical fatigue like neck and hip strains

\section{RESULTS}

The questionnaire study was conducted among 100 healthcare providers of IGIDS who have attended at least two treatment camps and have used the UBRD to provide dental care to the patients and 100 patients from the camp sites who have undergone treatment with the aid of UBRD. The 6-point questionnaire provided to the patients were in their regional language and their opinion was received after each of them successfully underwent their respective treatment form the healthcare provider. The data from healthcare providers who have worked with the UBRD in at least two camp sites were collected in the form a 11-point questionnaire. The obtained data were analyzed and the most relevant findings have been tabulated.

\section{From the Patient Perspective}

$91.8 \%$ of the population agreed that they underwent neck and hip strains while undergoing dental care with the UBRD which might have a shortcoming of the device and this would also imply that it would have been difficult for the patient to maintain the same neck position required by the dentist for a long duration and $8.2 \%$ did not experience any physical fatigue (Fig. 2). $22.4 \%$ of the population voted that they could cooperate well for treatment with the device as best as they could with the conventional dental chair. $62.3 \%$ of the population rated their level of cooperation above 5 on a scale of $1-10$ and $3 \%$ voted below 5 . A $12.2 \%$ of the population did not answer the question (Fig. 3).

From the perspective of the healthcare providers, $81.7 \%$ of the population under study agreed that they were able to provide better dental care for the patients at camp sites with the UBRD than without it (Fig. 4). 57.3\% have agreed that they experienced physical fatigue like neck and hip strain while working for long hours with the UBRD, 32.3\% have neither agreed or disagreed to this statement probably because they have not had the opportunity to work for a longer duration and $10.4 \%$ have not felt any physical fatigue (Fig. 5). Good accessibility to the operative site is a fundamental requirement to do a satisfactory treatment and $60.4 \%$ of the healthcare providers agreed that it is difficult to do extraction of posterior segments with the device and $16.7 \%$ did not find any difficulty (Fig. 6). 96.9\% of the healthcare providers agreed that the device is sufficient and useful to provide dental care in localities 


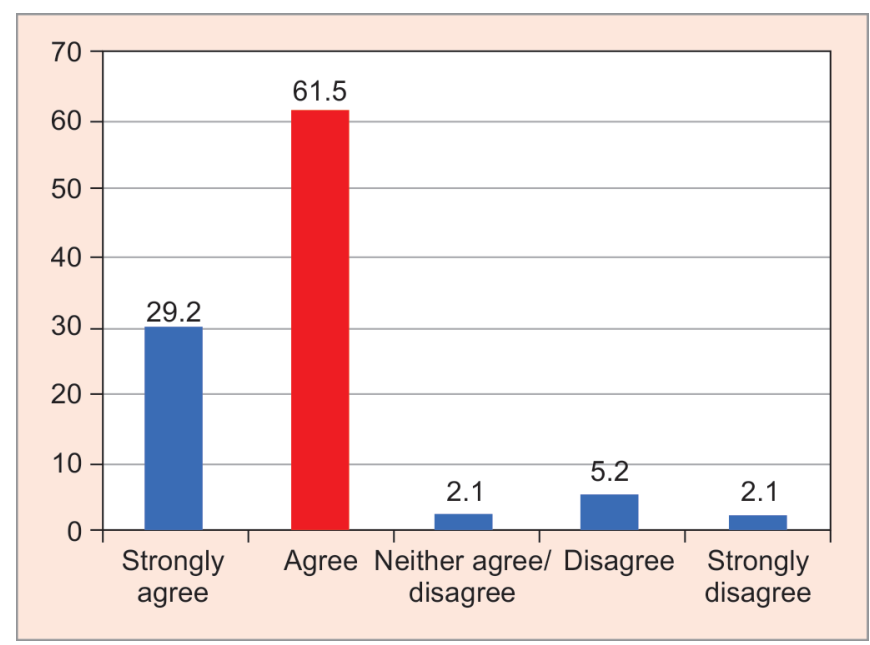

Fig. 4: Perception of the healthcare providers. You have been able to provide better dental care during camps with the UBRD than without it

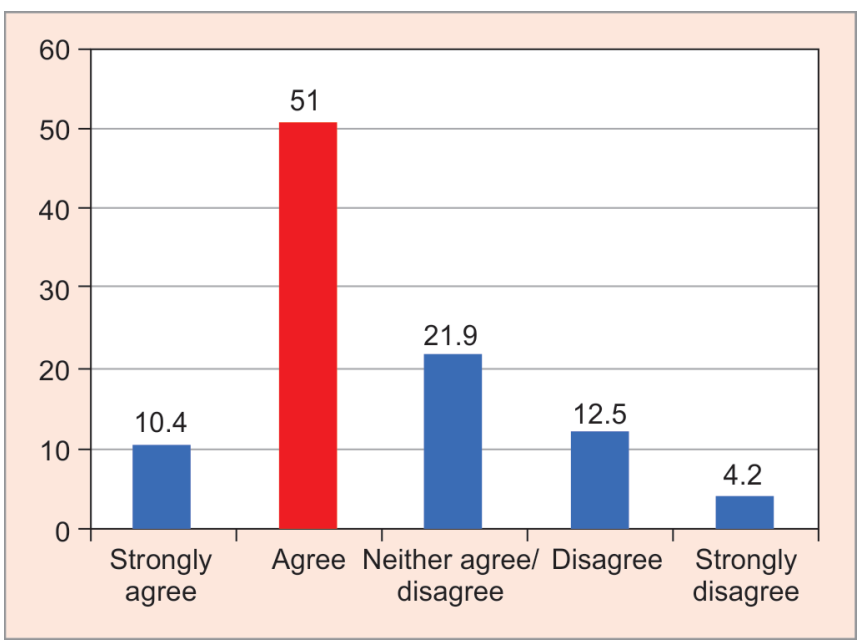

Fig. 6: Perception of the healthcare providers. You find difficulty in performing extraction of posterior segments

with limited access to resources and only $2.1 \%$ disagreed to this statement, which suggests that the device is useful and has helped increase the positive outcomes of camps (Fig. 7). Also, majority of the healthcare providers agreed that the UBRD would be sufficient to provide dental care in a PHC. This is a significant outcome of the study because it would considerably reduce the cost of equipment needed to provide dental care and this would lead to provide dental care to a larger population who might require treatment and visit the PHC. To evaluate the chances that certain dental procedures can be performed with an improved capability compared to others which might depend on the difficulty of the procedure or the sextant of the oral cavity that requires treatment, $76 \%$ of the healthcare providers suggested that ultrasonic scaling is could be performed with the best outcome with the use of the UBRD and $14.6 \%$ suggested that extraction can be performed with maximum efficiency and $7.3 \%$ voted for restorative procedures (Fig. 8). When asked to compare the ease of use and efficiency of the UBRD as compared to that of a conventional chair, $70.3 \%$ of the healthcare providers rated the UBRD above the range of 5 on a scale of 1 to 10 and $8.3 \%$ rated it below the range of 5 , which suggests that though

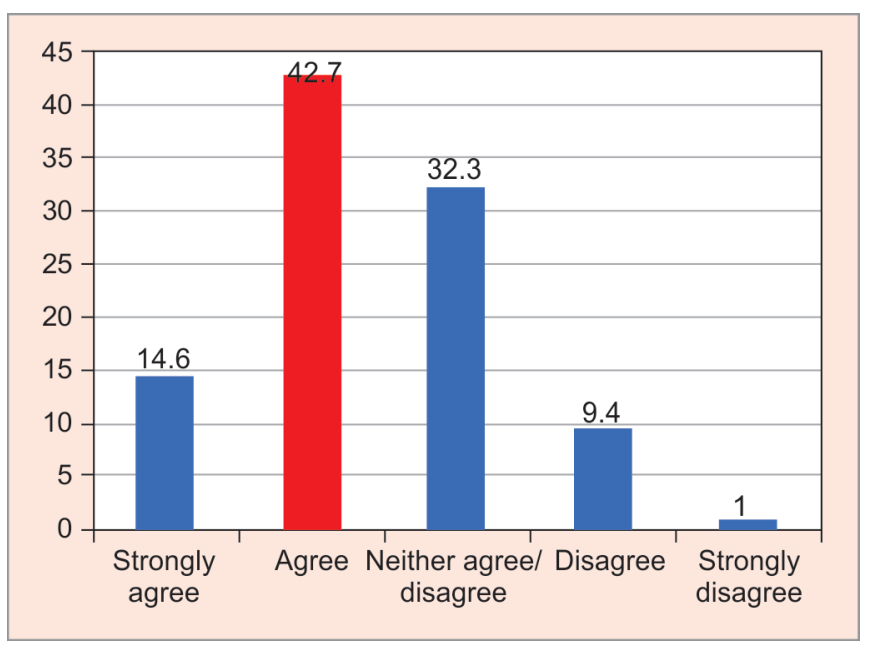

Fig. 5: Perception of the healthcare providers. While working with the device for long hours, physical fatigue like neck strains is experienced?

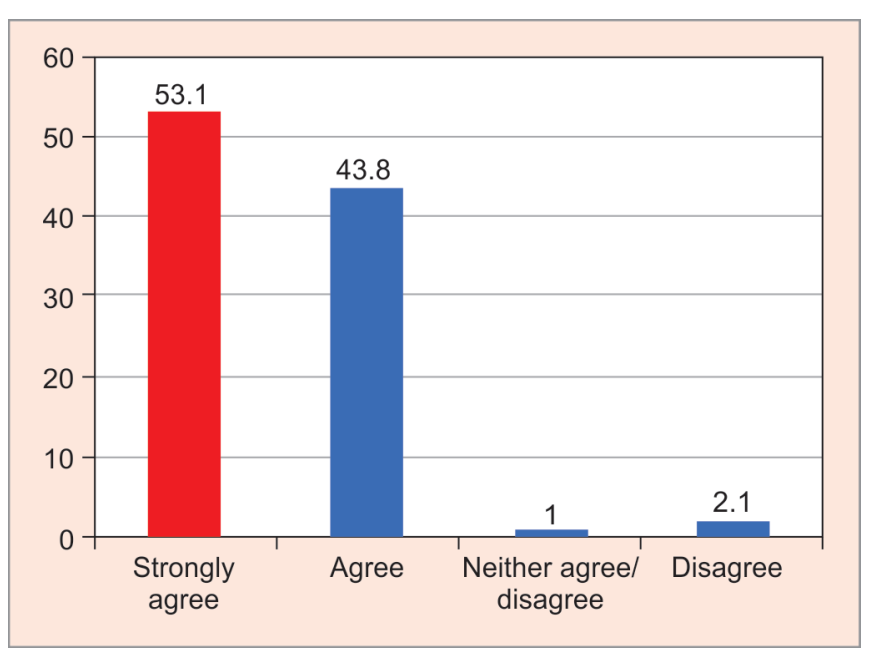

Fig. 7: Perception of the healthcare providers. Do you agree that to provide dental care in areas with less resources the device is very helpful?

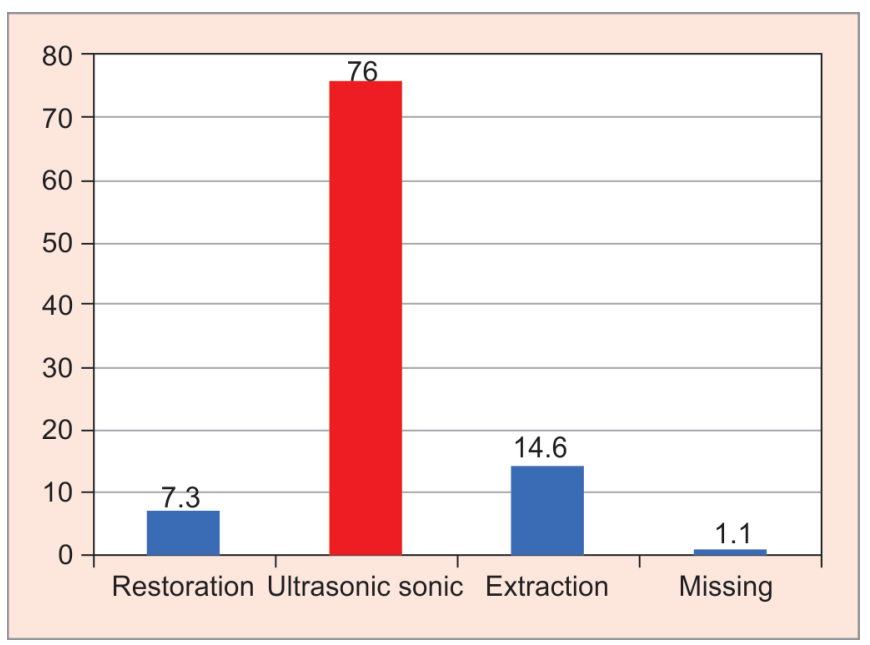

Fig. 8: Perception of the healthcare providers. Which of the following procedures do you think that can be performed best with the UBRD? 


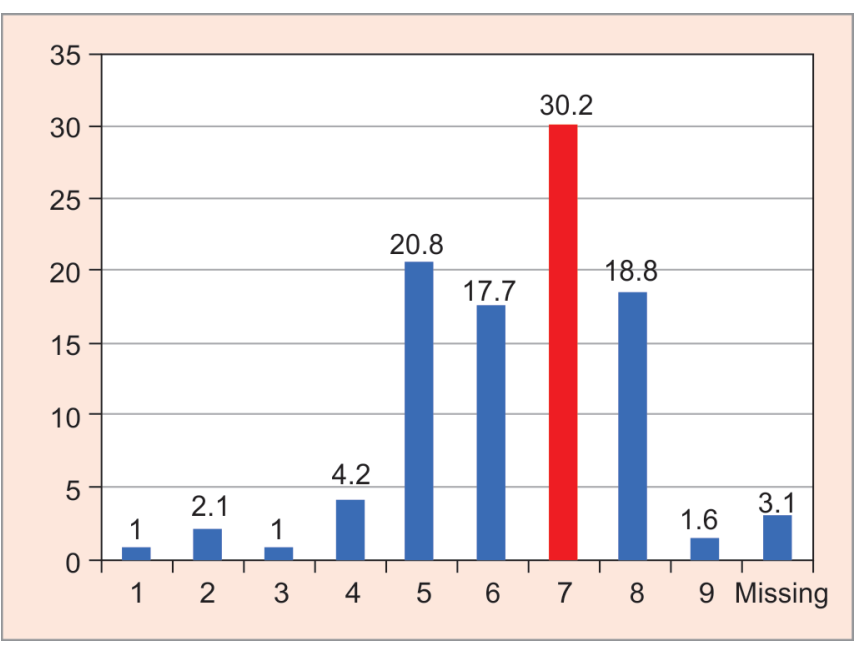

Fig. 9: Perception of the healthcare providers. How would you rate the efficiency of conventional dental chair to the UBRD device on a scale of $1-10$ ?

the device does not equal the efficiency of the conventional dental chair it is definitely the best option to use at camp sites where we have to work with limited resources (Fig. 9).

\section{Discussion}

Oral diseases remain one of the major global public health challenges, and the worldwide urban-rural disparities in oral health are significant. Residents in rural areas generally suffer from a higher prevalence and severity of dental caries and periodontal disease, yet they face numerous difficulties and barriers in accessing oral healthcare. Portable dental equipment has been defined as any non-facility in which dental equipment, utilized in the practice of dentistry, is transported to and utilized on a temporary basis at an out of office location including, but not limited to (a) another dentists' offices, (b) patients homes, (c) schools, (d) nursing homes, or (e) other institutions. Today, a variety of portable dental equipment is available that includes foldable dental chairs, hand held intraoral radiographic machines, suitcase or trolley units having attachments for ultrasonic scalers and airotor/micro-motor handpieces, high and low speed suction, built-in compressor systems, lathes and portable dark rooms. As an alternative to a portable dental chair, a device that called the UBRD has been developed, which aims to bridge the gap between the lack of adequate dental chairs and the increasing number of patients with oral healthcare needs and to also tend to the perceived difficulty of transporting them to a rural camp site. This study was undertaken to scrutinize the effects of UBRD, so that appropriate efforts could be taken to modify it for the better. Majority of the study population had undergone dental treatment before either in dental clinics or in camp sites with the aid of portable dental chairs and hence they could compare and validate their experiences accordingly. If this device proves to provide successful treatment, it can be either used as an alternative in a PHC. The healthcare providers suggested modifications that can be performed to the device to increase its efficiency and the most common suggestions were that the device would need more flexibility, height adjustment, and have an illumination device attached to it. From the data obtained, it can be unarguably agreed that the study population unanimously agreed that working with the UBRD was beneficial in providing acceptable treatment care to the patients.

Cost minimization of primary oral healthcare service delivery in rural areas appears to be achievable using portable dental units due to their lower fabrication and maintenance cost. But in situations where outreach treatment programs are aimed at providing dental care to a large group of people with very limited resources and in a very limited time frame, the UBRD has shown to benefit both the dentist and the patient to provide and receive healthcare efficiently.

The limitations of the study are that, when the data obtained was compiled it could be noticed that some of the questions were not understood properly by the patients and hence gave conflicting results. Second, it was noticed that some patients did not fill in the forms personally, which might be the reason of biased answers.

\section{Conclusion}

This study gives an insight as to the benefits of the UBRD and the areas where it needs improvement to increase its efficiency. The data obtained from the survey showed that the UBRD is beneficial in rendering improved quality dental care in areas with minimal resources and also could be used in PHCs. The study also throws limelight on the drawbacks of the device, that it causes physical fatigue to both the patient and the dentist while working for long hours. The inventors will focus further on making adjustments to the device to make it more comfortable to the patients and the healthcare providers.

\section{Clinical Significance}

This cross-sectional study was useful in evaluating the efficiency of the UBRD and gave a better understanding of the degree of help in rendered in making treatment care more acceptable and easy to provide from the view of a patient and healthcare provider.

\section{References}

1. Goel P, Goel A, Torwane NA. Cost-efficiency of indigenously fabricated mobile-portable dental unit. J Clin Diagnos Res 2014;8(7):ZC06-ZC09. DOI: $10.7860 / J C D R / 2014 / 8351.4534$.

2. Shaver RO. Differences between the traditional and the mobile extended care facility practice. J Colo Dent Assoc 1991;69(4):11-14.

3. Berkey DB, Gordon S. Memorial lecture. Current state of oral health care in institutionalized older adults. Spec Care Dentist 1996;16(4):143-146. DOI: 10.1111/j.1754-4505.1996.tb00849.x. 